\title{
Room temperature Peierls distortion in small diameter nanotubes
}

\author{
D. Connétable, ${ }^{1}$ G.-M. Rignanese, ${ }^{2,3}$ J.-C. Charlier ${ }^{2,3}$ and X. Blase ${ }^{1}$ \\ 1 Laboratoire de Physique de la Matière Condensée et des Nanostructures (LPMCN), \\ CNRS and Université Claude Bernard Lyon I, \\ Bâtiment Brillouin, 43 Bd du 11 Novembre 1918, 69622 Villeurbanne Cedex, France. \\ 2 Unité de Physico-Chimie et de Physique des Matériaux (PCPM), \\ Université Catholique de Louvain, 1 Place Croix du Sud, B-1348 Louvain-la-Neuve, Belgium \\ ${ }^{3}$ Research Center on Microscopic and Nanoscopic Electronic Devices and Materials (CERMIN), \\ Université Catholique de Louvain, B-1348 Louvain-la-Neuve, Belgium
}

(Dated: November 16, 2018)

\begin{abstract}
By means of $a b$ initio simulations, we investigate the phonon band structure and electron-phonon coupling in small 4 - $\AA$ diameter nanotubes. We show that both the $\mathrm{C}(5,0)$ and $\mathrm{C}(3,3)$ tubes undergo above room temperature a Peierls transition mediated by an acoustical long-wavelength and an optical $q=2 k_{F}$ phonons respectively. In the armchair geometry, we verify that the electron-phonon coupling parameter $\lambda$ originates mainly from phonons at $q=2 k_{F}$ and is strongly enhanced when the diameter decreases. These results question the origin of superconductivity in small diameter nanotubes.

PACS numbers: 61.46.+w, 61.50.Ah, 63.20.Kr, 63.22.+m, 68.35.Rh, 74.25.Kc, 74.70.Wz
\end{abstract}

Superconductivity (SC) has been recently discovered in the 4 - $\AA$ diameter carbon nanotubes (CNTs) embedded in a zeolite matrix [1] with a transition temperature $T_{S C}=15 \mathrm{~K}$, much larger than that observed in the bundles of larger diameter tubes [2]. This has stimulated a significant amount of work at the theoretical level to understand the origin of the SC transition [3, 4, 5, [6]. While both reduced screening and quantum/thermal fluctuations disfavor superconductivity in 1D systems, early work on fullerenes [7] and nanotubes [8] suggested that the curvature significantly enhances the strength of the electron-phonon (e-ph) coupling. This is consistent with the softening of the Raman modes under tube diameter reduction [9].

In 1D systems, the enhancement of the e-ph deformation potential should also favor the occurrence of Peierls, or charge density wave (CDW), transitions driving the system into an insulating phase. This possibility has been explored in early works on the basis of model Hamiltonians [10]. The strength of the e-ph coupling was either extrapolated from its value in graphite or taken as an adjustable parameter. The Peierls instability transition temperatures $T_{C D W}$ were estimated to be much smaller than room temperature: for instance, Huang et al. 10] find $T_{C D W} \sim 9.1 \mathrm{~K}$ for a $\mathrm{C}(5,5)$ tube. Further, long-wavelength optical 9] or acoustical [1] "opening the gap" phonon distortions have also been explored and the transition temperature was again predicted to be a few degrees Kelvin.

The Peierls transition in $4-\AA$ diameter CNTs has not been addressed explicitly, except for a recent tightbinding exploration of the $\mathrm{C}(5,0)$ tube 12 and an $a b$ initio study of the $\mathrm{C}(3,3)$ one [13]. While the inclusion of electronic screening in the non-self-consistent approach 12] for the $\mathrm{C}(5,0)$ case yields a negligible $T_{C D W}$, the $a b$ initio simulations predict for the $\mathrm{C}(3,3)$ tube a
$T_{C D W}$ temperature of $\sim 240 \mathrm{~K}$. These results suggest that the $\mathrm{C}(5,0)$ tube is the only candidate for a $\mathrm{SC}$ transition at $15 \mathrm{~K}$. However, such a large discrepancy between the two results call for a unified treatment of both nanotubes within a parameter-free approach.

In the present work, we study by means of $a b$ initio simulations the phonon band structure and e-ph coupling in 4 - $\AA$ diameter CNTs. We show that the $\mathrm{C}(5,0)$ and $\mathrm{C}(3,3)$ tubes both undergo a Peierls distortion with a critical temperature $T_{C D W}$ larger than room temperature. While the distortion is associated with a longwavelength acoustical mode in the $\mathrm{C}(5,0)$ case, the e-ph coupling is dominated by phonons at $q=2 k_{F}$ in the case of $\mathrm{C}(\mathrm{n}, \mathrm{n})$ nanotubes with a coupling parameter $\lambda$ which is strongly enhanced with decreasing diameter.

The electronic and vibrational properties are studied within a pseudopotential 14 planewave approach to density functional theory (DFT). We adopt the local density approximation (LDA) and a 50 Rydb cut-off to expand the electronic eigenstates. The phonon modes are calculated at arbitrary $q$-vectors using the perturbative DFT approach 15]. (in what follows $q$ - and $k$-vectors label phonon and electron momenta respectively). Special care is taken in sampling the electronic states around the Fermi level using an adaptative $k$-grid technique. The energy levels are populated using either a Gaussian broadening [16] or a Fermi-Dirac (FD) distribution within Mermin's generalization [17] of DFT to the canonical ensemble. The latter technique explicitly accounts for the effect of temperature. We use a periodic cell allowing for $10 \AA$ vacuum between the nanotubes.

We start by studying the nanotubes band structure after careful structural relaxation with a $25 \mathrm{meV}$ FD distribution broadening (T 300 K). The first important outcome of this calculation is the spontaneous zone-center deformation of the $\mathrm{C}(5,0)$ tube 18 . From the $\mathrm{D}_{10 h}$ sym- 


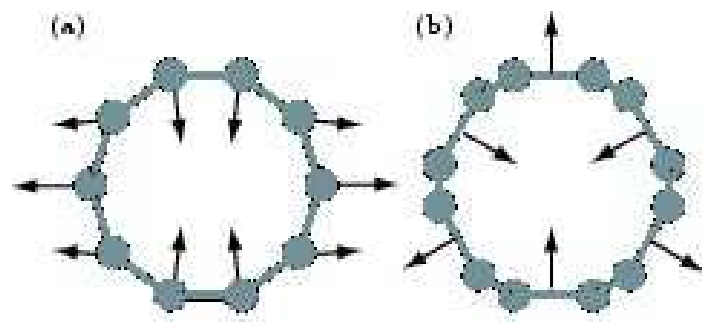

FIG. 1: Symbolic representations of the out-of-plane acoustical and optical modes at $\Gamma$ for the phonon bands driving the Peierls instability in the (a) $\mathrm{C}(5,0)$ and (b) $\mathrm{C}(3,3)$ tubes, respectively.

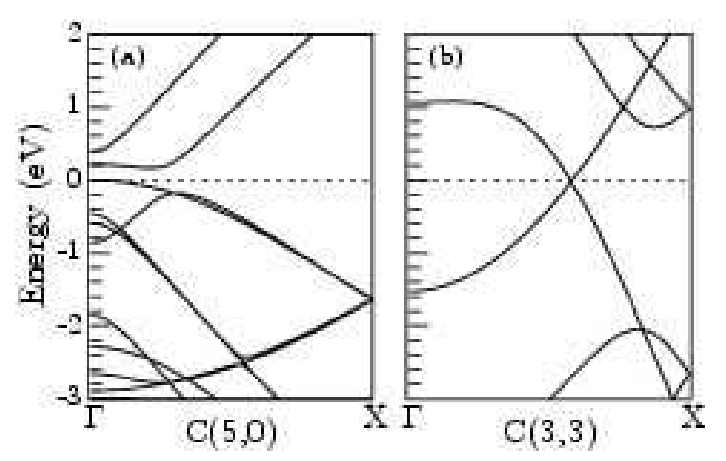

FIG. 2: Band structures of (a) the "distorted" $\mathrm{D}_{2 h} \mathrm{C}(5,0)$ nanotube and (b) the $\mathrm{C}(3,3)$ tube. The zero of energy has been set to the top of the valence bands and at the Fermi level, respectively.

metry, the structure relaxes to form an elliptic tube $\left(\mathrm{D}_{2 h}\right.$ symmetry), as indicated by the arrows in Fig. 1(a), with principal axes of $3.83 \AA$ and $4.13 \AA$.

The corresponding band structure is provided in Fig. 2(a). As a result of the reduction of symmetry, the repulsion of the hybridized $\sigma-\pi$ bands [19] at the Fermi level opens $\mathrm{a} \sim 0.2 \mathrm{eV}$ band gap (LDA value). This implies that the $C(5,0)$ nanotube undergoes a metallicsemiconducting transition with $T_{C D W}$ larger than room temperature. Following Ref. 9 , such a transformation can be referred to as a "pseudo-Peierls" transition since it opens the band gap through a (long-wavelength) structural deformation.

In the $\mathrm{C}(3,3)$ case, we do not observe any zone-center deformation leading the band structure away from the well-documented [20] zone-folding picture of two linear bands crossing at the Fermi level $E_{F}$. However, compared to the zone-folding analysis, the Fermi wavevector $k_{F}$ is no longer at $2 / 3 \Gamma \mathrm{X}$ but at $\sim 0.58 \Gamma \mathrm{X}$ so that $2 \pi / 2 k_{F}$ is no longer commensurate with the unperturbed lattice (or commensurate with a very large supercell). This is a well documented effect of the curvature.

We further present in Fig. 31(a) the phonon band structure of the $\mathrm{C}(3,3)$ tube $[21]$. The most salient feature is

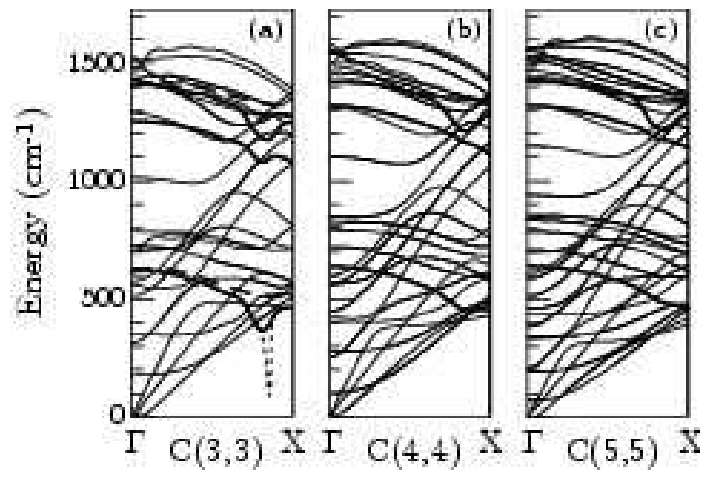

FIG. 3: Phonon band structures for the $\mathrm{C}(\mathrm{n}, \mathrm{n})$ nanotubes with $\mathrm{n}=3,4$, and 5 from left to right obtained with a Gaussian broadening of $136 \mathrm{meV}$ (10 mRy). The dotted line for the $\mathrm{C}(3,3)$ nanotube corresponds to the additional explicit calculation of the phonon modes around $q=2 k_{F}$ with a reduced $68 \mathrm{meV}$ ( $5 \mathrm{mRy}$ ) broadening. Important bands appear as thicker lines. The "undressed" $\omega_{q}^{0}$ bands (see text) are indicated with thick dashed lines.

the giant Kohn anomaly [25] at $q=2 k_{F}$ leading to a dramatic softening of a few phonon modes 26. In particular, the optical band starting at $620 \mathrm{~cm}^{-1}$ at $\Gamma$ becomes the lowest vibrational state at $q=2 k_{F}$ [thick/dotted low lying band in Fig. 3(a)]. The associated atomic displacements, indicated in Fig. 1(b), correspond to an outof-plane optical mode in agreement with the results of Ref. 12. This is consistent with the analysis provided by Mahan [27]: the coupling of electrons with out-of-plane optical modes should be enhanced away from zone-center and with decreasing diameter. The predominance of such a mode for e-ph coupling is a strong manifestation of the importance of curvature in small diameter tubes.

The value of the associated frequency at $q=2 k_{F}$ strongly depends on the energy distribution broadening used in the phonon calculation, as clearly illustrated in Fig. 3(a) by the difference between the thick and dotted lines. Using a $25 \mathrm{meV}$ FD distribution (T $300 \mathrm{~K}$ ), we find a negative phonon mode $\omega_{2 k_{F}, \nu=1}$ of $-200 \mathrm{~cm}^{-1}$. This means that the $C(3,3)$ tube undergoes a Peierls distortion with $T_{C D W}$ larger than room temperature [2d]. Consequently, at the mean-field $S C$ transition temperature measured for the 4-A diameter CNTs $\left(T_{S C}=15 \mathrm{~K}\right)$, the $C(5,0)$ and $C(3,3)$ tubes should both be semiconducting, seriously questioning the origin of superconductivity in small diameter tubes.

Our results are in good agreement with those of Ref. 13 concerning the $\mathrm{C}(3,3)$ tube, but contrast significantly with those based on a model for the interaction Hamiltonians and the electronic susceptibility 12 . We emphasize that our self-consistent treatment automatically includes the renormalization of both the e-ph and e-e interactions by electronic screening at the DFT level.

To study the influence of tube diameter on the soften- 
ing of the modes at $q=2 k_{F}$, we represent in Fig. 3(b,c) the phonon band structure of the $\mathrm{C}(\mathrm{n}, \mathrm{n})$ tubes with $\mathrm{n}=4$ and 5 21]. Again, we observe that the same optical band (thick low lying band) is softened at $2 k_{F}$, but clearly the effect is strongly reduced with increasing diameter. This confirms that $\mathrm{T}_{C D W}$ will quickly lower with increasing diameter. The large amount of $s p^{3}$ character in ultrasmall tubes invite to draw a comparison with the SC transition in doped carbon clathrates 22] and diamond 23].

Surprisingly, the softening of another mode at higher energy (starting as an optical mode around $1450 \mathrm{~cm}^{-1}$, also indicated by a thick line) seems to be less sensitive to the diameter. This softening is the signature of a large e-ph coupling which might thus play an important role in the transport properties of larger CNTs. 24

To further quantify the strength of the e-ph interaction, we can calculate the e-ph coupling matrix elements $g_{q \nu}\left(k n n^{\prime}\right)$ and the coupling constant $\lambda$ :

$$
\begin{aligned}
& \lambda=\sum_{q, \nu} \lambda_{q, \nu}=2 N\left(E_{f}\right) \sum_{q, \nu}<\left|g_{q \nu}\right|^{2}>/ \hbar \omega_{q \nu} \\
& <\left|g_{q \nu}\right|^{2}>=\int \frac{d k}{L_{B Z}} \sum_{n, n^{\prime}}\left|g_{q \nu}\left(k n n^{\prime}\right)\right|^{2} \frac{\delta\left(\epsilon_{k+q, n^{\prime}}\right) \delta\left(\epsilon_{k n(\boldsymbol{q}}\right)}{N\left(E_{F}\right)^{2}} \\
& g_{q \nu}\left(k n n^{\prime}\right)=\left(\frac{\hbar}{2 M \omega_{q \nu}}\right)^{1 / 2}<\psi_{n \mathbf{k}}^{0}\left|\hat{\epsilon}_{\mathbf{q} \nu} \cdot \frac{\delta V}{\delta \hat{u}_{q \nu}}\right| \psi_{n^{\prime} \mathbf{k}+\mathbf{q}}^{0}(ß)
\end{aligned}
$$

Due to conservation of energy and momentum, only vibrational states around $q=\Gamma$ and $\pm 2 k_{F}$ contribute to $\lambda$, as the nesting factor 29] n(q) quickly decays away from these points. At $\Gamma$, only four modes contribute to $\lambda$ due to symmetry considerations. They are the radial breathing mode (RBM), the optical out-of-plane (ZO) mode, and the in-plane optical longitudinal $\mathrm{A}_{1}(\mathrm{~L})$ and transverse $A_{1}(\mathrm{~T})$ (G-band) modes (see Ref. 9) located respectively at $536 \mathrm{~cm}^{-1}, 692 \mathrm{~cm}^{-1}, 1354 \mathrm{~cm}^{-1}$ and $1431 \mathrm{~cm}^{-1}$ in the $\mathrm{C}(3,3)$ tube.

In Table I we report the parameter $\lambda(\nu, q \subset\{\Gamma\})$ defined as the $q$-sum of all the $\lambda_{q, \nu}$ (see Eq. 1) for $q$ in the neighborhood of $\Gamma$ on a given $\nu$-band [30]. This coupling strength varies from one band to another and is enhanced when the tube diameter decreases. However, the variation with the diameter differs from one mode to another and it is difficult to extract a simple scaling law.

We now discuss the e-ph coupling at $q=2 k_{F}$. The strong variation of the lowest frequency $\omega_{2 k_{F}, \nu=1}$ with temperature raises the question of the meaning of $\lambda_{2 k_{F}, \nu=1}$ calculated with the $g_{q \nu}$ and $\omega_{q \nu}$ values obtained from ab initio calculations. The difficulties have been clearly exposed in Ref. 12. If one is interested in studying the SC transition, one faces the difficulty that at the experimental $\mathrm{T}_{S C}$, the self-consistent $g_{2 k_{F}, \nu}$ and $\omega_{2 k_{F}, \nu}$ values are those of the $\mathrm{CDW}$ phase. As $T_{C D W}$ is larger than any realistic SC transition temperature, we will not attempt to estimate $\mathrm{T}_{S C}$ with e-ph vertices and phpropagator properly "undressed" from the CDW instability [12]. We limit ourselves to identifying the relevant
TABLE I: Contributions $\left(\lambda_{\nu}\right)$ to the coupling constant for all the $q$-vectors in the neighborhood of $\Gamma(q \subset\{\Gamma\})$ and $2 k_{F}$ $\left(q \subset\left\{2 k_{F}\right\}\right)$ for the $\mathrm{C}(\mathrm{n}, \mathrm{n})$ tubes with $\mathrm{n}=3,4$, and 5 . The corresponding frequencies $\left(\omega_{\nu}\right)$ at $\Gamma$ and $2 k_{F}$ are indicated in $\mathrm{cm}^{-1}$. For the $\mathrm{C}(3,3)$ tube, the values underlined for $q \subset$ $\left\{2 k_{F}\right\}$ correspond to the "undressed" frequencies (see text). The global coupling constant $(\lambda)$ is indicated at the last line.

\begin{tabular}{rrrrrrr}
\hline \hline & \multicolumn{2}{c}{$\mathrm{C}(3,3)$} & \multicolumn{2}{c}{$\mathrm{C}(4,4)$} & \multicolumn{2}{c}{$\mathrm{C}(5,5)$} \\
& $\omega_{\nu}$ & \multicolumn{1}{c}{$\lambda_{\nu}$} & \multicolumn{1}{c}{$\omega_{\nu}$} & $\lambda_{\nu}$ & $\omega_{\nu}$ & $\lambda_{\nu}$ \\
\hline$q \subset\{\Gamma\}$ & & & & & & \\
$\mathrm{RBM}$ & 536 & 0.009 & 416 & 0.005 & 338 & 0.001 \\
$\mathrm{ZO}$ & 692 & 0.009 & 799 & 0.004 & 848 & 0.001 \\
$\mathrm{~A}_{1}(\mathrm{~L})$ & 1354 & 0.007 & 1468 & 0.009 & 1538 & 0.002 \\
$\mathrm{~A}_{1}(\mathrm{~T})$ & 1431 & 0.012 & 1505 & 0.009 & 1570 & 0.002 \\
$q \subset\left\{2 k_{F}\right\}$ & & & & & & \\
& $\underline{520}$ & $\underline{0.074}$ & 414 & 0.022 & 497 & 0.010 \\
& $\underline{1920}$ & $\underline{0.026}$ & 509 & 0.010 & 533 & 0.004 \\
& $\underline{1144}$ & $\underline{0.018}$ & 1013 & 0.010 & 1154 & 0.009 \\
& & 0.156 & 1180 & 0.014 & 1237 & 0.011 \\
\hline \hline
\end{tabular}

modes and providing the values for $\lambda_{2 k_{F}, \nu=1}$ obtained above $T_{C D W}$, which are those that matter for transport measurements in the normal state.

While the $g_{2 k_{F}}$ parameters are rather stable with temperature above $T_{C D W}$, the phonon frequencies still vary significantly. Following Ref. 12, we provide an upperbound for $\omega_{2 k_{F}, \nu}$ by removing the Kohn's anomaly. Namely, we assume that around $q=2 k_{F}$,

$$
\omega_{q, \nu}^{2}=\left(\omega_{q, \nu}^{0}\right)^{2}+A \times \ln \left|\left(q-2 k_{F}\right) /\left(q+2 k_{F}\right)\right|
$$

and fit the $\omega_{q, \nu}^{2}$ bands by a cubic polynomial for $\left(\omega_{q, \nu}^{0}\right)^{2}$ plus the logarithmic term that we subtract to obtain the bare phonon frequency. The important "undressed" bands (corresponding to $\omega_{q, \nu}^{0}$ ) are represented by dashed lines in Fig. 3(a). The related frequencies and $\lambda_{2 k_{F}, \nu}$ values are underlined in Table @ This treatment is applied to both states presenting the largest Kohn anomaly, but its effect is clearly more pronounced for the low-lying $\nu=1$ band.

Using the $\omega_{2 k_{F}, \nu}^{0}$ frequencies leads to providing a lower bound for the corresponding $\lambda_{2 k_{F}, \nu}$ e-ph parameter. Even in that limit, we observe that the coupling with modes at $q=2 k_{F}$ is significantly larger than with long-wavelength phonons. As at zone-center, few modes with $q=2 k_{F}$ couple to the electrons and the strength of the coupling is significantly enhanced with decreasing diameter. In the $\mathrm{C}(3,3)$ case, we obtain in the undistorted phase a density of states $N\left(E_{F}\right)=0.4$ states $/ \mathrm{eV}$ cell-spin, yielding an e-ph potential $V^{e p}=\lambda / N\left(E_{F}\right)=$ $440 \mathrm{meV}$. This is larger than that in the fullerenes (60$70 \mathrm{meV}$ ), but it is not sufficient to lead to any significant $T_{S C}$ value. The much larger density of states in the " $D_{10 h}$ " $\mathrm{C}(5,0)$ tube $\left(N\left(E_{F}\right)=1.8\right.$ states/eV-cell-spin) 
may lead in principle to a large $T_{S C}$ value. However, as shown above, the prevailing structure around the experimental $T_{S C}$ temperature is the insulating $D_{2 h}$ one.

In conclusion, we have shown that the "metallic" $\mathrm{C}(5,0)$ and $\mathrm{C}(3,3)$ nanotubes undergo a Peierls transition with a critical temperature larger than $300 \mathrm{~K}$. Our results are derived within Mermin's generalization of DFT to finite temperature systems and do not rely on models for the e-ph interactions nor the electronic susceptibility. Additional physical ingredients (interaction with the zeolite network, possible defective structure, Luttinger liquid character, etc.) might reconcile these results with the experimentally observed $\mathrm{SC}$ transition at $\mathrm{T}_{S C}=15 \mathrm{~K}$. Acknowledgements: Calculations have been performed at the French CNRS national computer center at IDRIS (Orsay). GMR and JCC acknowledge the National Fund for Scientific Research [FNRS] of Belgium for financial support. Parts of this work are also connected to the Belgian Program on Interuniversity Attraction Poles (PAI5/1/1) on Quantum Size Effects in Nanostructured Materials, to the Action de Recherche Concertée "Interaction électron-phonon dans les nanostructures" sponsored by the Communauté Française de Belgique, and to the NanoQuanta and FAME European networks of excellence.

[1] Z.K. Tang et al., Science 292, 2462 (2001).

[2] M. Kociak et al., Phys. Rev. Lett. 86, 2416 (2001).

[3] J. González, Phys. Rev. B 87, 136401 (2001); ibid., Phys. Rev. Lett. 88, 076403 (2002); J.V. Alvarez and J. González, Phys. Rev. Lett. 91, 076401 (2003).

[4] A. Sédéki, L.G. Caron, C. Bourbonnais, Phys. Rev. B 65, 140515(R) (2002).

[5] K. Kamide, T. Kimura, M. Nishida, and S. Kuhihara, Phys. Rev. B 68, 024506 (2003).

[6] A. De Martino and R. Egger, Phys. Rev. B 67, 235418 (2003).

[7] M. Schluter, M. Lannoo, M. Needels, G. A. Baraff, and D. Tománek Phys. Rev. Lett. 68, 526 (1992).

[8] L.X. Benedict, V.H. Crespi, S.G. Louie, and M.L. Cohen, Phys. Rev. B 52, 14935 (1995).

[9] O. Dubay, G. Kresse, and H. Kuzmany, Phys. Rev. Lett. 88, 235506 (2002).

[10] J.W. Mintmire, B.I. Dunlap, and C.T. White, Phys. Rev. Lett. 68, 631 (1992); R. Saito, M. Fujita, G. Dresselhaus, M.S. Dresselhaus, Phys. Rev. B 46, 1804 (1992); K. Harigaya, M. Fujita, Phys. Rev. B 47, 16563 (1993); Y. Huang et al., Solid State Commun. 97, 303 (1996); K. Tanaka et al., Int. J. Quan. Chem. 63, 637 (1997).

[11] M.T. Figge, M. Mostovoy, and J. Knoester, Phys. Rev. Lett. 86, 4572 (2001); ibid., Phys. Rev. B 65, 125416
(2002).

[12] R. Barnett, E. Demler, E. Kaxiras, cond-mat/0407644 ibid., cond-mat/0305006

[13] K.-P. Bohnen et al., Phys. Rev. Lett. 93, 245501 (2004); Phys. Rev. Lett. 93, 259901(E) (2004). Paper published after submission of our manuscript.

[14] N. Troullier and J.L. Martins, Phys. Rev. B 43, 1993 (1991).

[15] S. Baroni, S. de Gironcoli, A. Dal Corso, and P. Giannozzi, Rev. Mod. Phys. 73 (2), 515 (2001); S. Baroni, A. Dal Corso, S. de Gironcoli, and P. Giannozzi, http://www.pwscf.org

[16] M. Methfessel and A. Paxton, Phys. Rev. B 40, 3136 (1989)

[17] N. Mermin, Phys. Rev. 137, A1441 (1965).

[18] This deformation occurs for small enough a FD energy broadening. This explains why it has not been reported so far. The present results have been obtained independently with the ABINIT (http://www.abinit.org) and PWSCF (http://www.pwscf.org) codes.

[19] X. Blase, L.X. Benedict, E.L. Shirley, S.G. Louie, Phys. Rev. Lett. 72, 1878 (1994).

[20] M. Machón, S. Reich, C. Thomsen, D. Sánchez-Portal, and P. Ordejón, Phys. Rev. B 66, 155410 (2002); H.J. Liu, and C.T. Chan, Phys. Rev. B 66, 115416 (2002).

[21] The phonon band structures are obtained by a special interpolation technique 15] based on the explicit calculation of the dynamical matrices on $20 q$-point grid.

[22] H. Kawaji et al., Phys. Rev. Lett. 74, 1427 (1995); K. Tanigaki et al., Nat. Mater. 2, 653 (2003); D. Connétable et al., Phys. Rev. Lett. 91, 247001 (2003).

[23] E.A. Ekimov et al., Nature (London) 428, 542 (2004); X. Blase et al., Phys. Rev. Lett. 93, 237004 (2004); L. Boeri et al., ibid., 237002 (2004); K.W. Lee and W.E. Pickett, ibid., 237003 (2004); H.J. Xiang et al., Phys. Rev. B 70, 212504 (2004).

[24] The "large radius" limit (graphite) has been addressed in: S. Piscanec et al., Phys. Rev. Lett. 93, 185503 (2004).

[25] W. Kohn, Phys. Rev.Lett. 2, 393 (1959).

[26] As expected, this softening does not appear in classical lattice dynamical models. See: Z.M. Li et al., Appl. Phys. Lett. 84, 4101 (2004). Our results do not exclude the existence at very low temperature of an acoustic-phononmediated instability [6, 11], but such an instability will be clearly dominated by the one at $q=2 k_{F}$.

[27] G.D. Mahan, Phys. Rev. B 68, 125409 (2003).

[28] Using various values of $\mathrm{FD}$ broadening, $\mathrm{T}_{C D W}$ can be estimated to lie with $300 \mathrm{~K}$ and $400 \mathrm{~K}$.

[29] $n(q)$ can be obtained by setting the $g$ coefficients to one in Eq. 2. It is a measure of "phase space availability" for electrons to be scattered by phonons of wavevector $q$.

[30] The domain of integration is defined by the extent of $n(q)$ around $\Gamma$. We have observed that $\lambda(q, \nu) / n(q)$ is nearly independent of $\mathrm{q}$ in this domain. This greatly helps in calculating $\lambda$ as $n(q)$ can be calculated with arbitrary precision by fitting the electronic bands around $\pm k_{F}$. 